Simone Müller*, Steven Hill, Sukwi Kim, Francis Müller, Nahoko Suzuki und Andres Toledo

\title{
Aufzeichnungen über einen unheimlichen Mönch: Eine kommentierte Übersetzung
}

https://doi.org/10.1515/asia-2019-0006

\section{Records about a Creepy Monk}

Abstract: Yōsōki (Records about a Creepy Monk, 1902) is a lesser-known short story of Izumi Kyōka, one of the most popular writers of the Meiji period. Kyōka, known for his fantastic, pictorial and folksy stories, belonged to the renowned literary circle Kenyūsha, founded by Ozaki Kōyō, that committed itself to light fiction and firmly rejected didactically motivated literature. The story Yōsōki presented here for the first time in German translation, stands prototypically in the tradition of the fantastic-aesthetic literature characteristic for Kyōka's writing.

Keywords: Izumi Kyōka, fantastic literature (gensō bungaku), literature of the Meiji period

Schlagwörter: Izumi Kyōka, Fantastik (gensō bungaku), Meiji-Literatur

\section{Einleitung}

Izumi Kyōka 泉鏡花 (1873-1939), 1873 in Kanazawa geboren, gilt als einer der populärsten Schriftsteller der Meiji-Zeit. In seinen zahlreichen Werken - die meisten davon Kurzgeschichten - verbinden sich Imagination, Volkstümlichkeit und eine eigentümliche Sprache zu einer künstlerischen Fertigkeit von hoher Perfektion, was ihm unter anderem das Attribut "Handwerker der Literatur" (bungei no aruchisan) ${ }^{1}$ eingebracht hat. Mishima Yukio 三島由紀夫 (1925-1970), ein dezidierter Verehrer von Izumi Kyōka, bezeichnete ihn gar als einzig wahres Genie der japanischen Literatur und setzte ihn hinsichtlich der Reinheit seines Romantizismus auf eine

1 Miyoshi 1980: 8.

*Corresponding author: Simone Müller, Universität Zürich, Asien-Orient-Institut, Zürichbergstrasse 4, 8032 Zürich, Schweiz. E-mail: simone.mueller@aoi.uzh.ch

Steven Hill, Sukwi Kim, Francis Müller, Nahoko Suzuki, Andres Toledo, Universität Zürich, Asien-Orient-Institut, Zürichbergstrasse 4, 8032 Zürich, Schweiz 
Stufe mit E.T.A Hoffmann (1776-1822). ${ }^{2}$ Kyōkas Literatur, so Mishima, repräsentiere die Meisterhaftigkeit eines weiblichen, dionysischen Stils. ${ }^{3}$ Tanizaki Jun'ichirō 谷崎 潤一郎 (1886-1965), ebenfalls ein Bewunderer Kyōkas, lobte die “unverfälschte japanische" Qualität seiner Werke. ${ }^{4}$

Bezeichnenderweise stammt Izumi Kyōka aus einer bekannten Handwerkerund Künstlerfamilie: der Vater war ein Ziseleur, der Bruder der Mutter ein NōSchauspieler. Diese familiären Umstände sollen das literarische Schaffen von Kyōka wesentlich geprägt haben. ${ }^{5}$ Eine weitere, vielbetonte Charakteristik von Izumi Kyōkas Literatur, namentlich sein Frauenbild, scheint ebenfalls Wurzeln in seiner Biographie zu haben: Er verlor bereits im zarten Alter von neun Jahren die Mutter, was in ihm eine tiefe Sehnsucht nach mütterlicher Geborgenheit und Liebe hinterliess, die sich in zahlreichen seiner Werke widerspiegelt.

Izumi Kyōka strebte bereits früh eine Schriftstellerkarriere an. Er fühlte sich insbesondere zu den Werken von Ozaki Kōyō 尾崎紅葉 (1847-1903) hingezogen und begab sich deshalb im Jahr 1891 nach Kyōto, um der 1885 ins Leben gerufenen literarischen Vereinigung Kenyūsha 硯友社 (Freunde des Tuschreibsteins), Kōyōs Literaturzirkel, beizutreten. Die einflussreiche Gruppierung war der Unterhaltung verpflichtet und lehnte didaktisch und intellektuell motivierte Literatur dezidiert ab. ${ }^{6}$ Auch Kyōka blieb zeitlebens der unterhaltenden Literatur verpflichtet und vermochte sich nie für den damals aufkommenden Naturalismus (shizenshugi bungaku) zu erwärmen, denn, so Kyōka, die Literatur sei kein Produkt des Intellekts, sondern des Gefühls (bungei wa dō shitatte chishiki no sanbutsu de wa nai, kanjō no sanbutsu de aru). ${ }^{7}$

Im Jahr 1893 debütierte Kyōka mit dem Fortsetzungsroman Kanmuri Yazaemon 冠弥左衛門 (Der gekrönte Yazaemon). Zwei Jahre später veröffentlichte er in der damals renommiertesten Literaturzeitschrift Bungei kurabu (Kunstklub) die beiden Erzählungen Yakō junsa 夜行巡査 (Der Nachwächter, 1895) und Gekashitsu 外科室 (Das Operationszimmer, 1895), was ihn auf einen Schlag ins Rampenlicht der Literaturszene rückte. In der Folge entwickelte sich Kyōka zusehends zu einem Erfolgsautor, dessen Popularität diejenige seines Lehrmeisters Kōyō sogar noch übertraf. Es folgten weitere Erfolgsromane wie Kōya hijiri 高野聖 (Der Heilige vom Berg Kōya, 1900) und Uta andon 歌行燈 (Lied der Laterne, 1910). Anfang des 20. Jahrhunderts, mit der wachsenden Popularität der naturalistischen Literatur in Japan, geriet Izumi

2 Mishima 1976, zit. nach Inouye 1996: 6.

3 Mishima 1975, zit. nach Cornyetz 1998: 112.

4 Tanizaki 1968, zit. nach Inouye 1996: 6.

5 Sasahara 1984.

6 Keene 1984: 119-120.

7 Izumi 1942 [1909]: 723. 
Kyōka zwar etwas aus dem Blickfeld, oder, in Tanizakis Worten: Er wurde "zurückgelassen und vergessen." 8 Er blieb seinem Stil jedoch treu und verfasste bis $\mathrm{zu}$ seinem Todesjahr 1939 zahlreiche weitere der Unterhaltung und der l'art pour l'art ${ }^{9}$ verpflichtete Werke, die sich bis heute einer breiten Leserschaft erfreuen.

Izumi Kyōkas Literatur wird gemeinhin als mystisch, symbolistisch und volkstümlich bezeichnet. Er ist zudem bekannt für seine schwer zugängliche, archaische Prosa. ${ }^{10}$ Er gilt deshalb sowohl als Traditionalist - er war massgeblich von der Edozeitlichen gesaku-Literatur beeinflusst und repräsentiert deshalb die vormoderne japanische Ästhetik ${ }^{11}$ - als auch als Stilist und bildet somit eine Brücke zwischen der Literatur der Vormoderne und der Moderne. ${ }^{12}$ Viele von Izumi Kyōkas Erzählungen enthalten makabre und fantastische Elemente - er hatte eine Vorliebe für Geistergeschichten ${ }^{13}$-, weshalb er gemeinhin dem Genre der so genannten gensō bungaku (fantastische Literatur) zugeordnet wird. Diese teilt, wie Henry J. Hughes herausstreicht, mit der westlichen Schauerliteratur eine Untergrabung religiöser und sozialer Normen, eine Obsession mit Sex und Tod sowie eine Angst vor dem Unbekannten. ${ }^{14}$ Kyōka wird deshalb zuweilen auch mit Autoren der europäischen Fantastik verglichen, etwa mit Gérard de Nerval (1808-1855), ${ }^{15}$ Autor des bekannten Romans Aurélia, ou le rêve et la vie (1855), in welchem sich Traum und Wirklichkeit formvollendet verbinden. Waki Akiko charakterisiert Kyōkas Erzählungen denn auch bezeichnenderweise als "Traumerzählungen" (yumegatari). ${ }^{16}$ Wie Mark Cody Poulton betont, verwendet Kyoka indes die Fantasie weniger als Flucht vor der Realität, sondern als Kritik der modernen Welt. ${ }^{17}$ Viele seiner Werke sind geprägt durch ein Spannungsverhältnis zwischen Zivilisation und Wildnis, die, so Poulton, Kyōkas Missbehagen mit den Effekten der Modernisierung zum Ausdruck bringen. Das Alte, Natürliche stehe hier gleichsam als positives Gegenbild zum Neuen und Zivilisierten. ${ }^{18}$ Allerdings war bei Kyōka auch die Natur nicht die unschuldige Idylle, sondern ein monströser Ort, in welchem die Natur dämonisiert, personifiziert und erotisiert, oder, in Poultons Terminologie, “animisiert” wird. ${ }^{19}$ Die Metamorphose

8 Tanizaki 1968: 336, zit. nach Inouye 1996: 7.

9 Miyoshi 1980: 12.

10 Cornyetz 1998: 104-105.

11 Cornyetz 1998: 104.

12 Inouye 1996: 8.

13 Cornyetz 1998: 104-105.

14 Hughes 2000: 60.

15 Waki 1974, zit. nach Poulton 1995a: 72.

16 Waki 1980: 96.

17 Poulton 1995a: 73.

18 Poulton 1995a: 88-89.

19 Poulton 1995a: 89. 
und das Dämonenhafte in Kyōkas Literatur, so Charles Shirō Inouye, sei Ausdruck einer "Aufrichtigkeit der Angst, die auf dem Grund von Kyōkas Ästhetik liegt.”20

Die zweite, bereits erwähnte Charakteristik von Izumi Kyōkas Literatur, sein Frauenbild, ist Gegenstand zahlreicher Studien. ${ }^{21}$ Bei Kyōka werden die Frauen als Göttinnen überzeichnet, die ihre eigene und ihrer Mitmenschen Gestalt nach Willkür verändern können. ${ }^{22}$ Sie sind oft "gefährlich" und "betörend" und dienen als Medium für vergangene und imaginierte Welten. ${ }^{23}$ Viele seiner Erzählungen beschreiben zudem offenherzig seine Muttersehnsucht (haha koi) und willensschwache Männer, die sich der Liebe und des Schutzes starker Frauen anbequemen. ${ }^{24}$ Wie Poulton aufzeigt, repräsentiert die "Mutter" in Kyōkas Literatur nicht eine Person, sondern weit eher eine Idee. ${ }^{25}$ Bezeichnenderweise machte Izumi zwei grosse übernatürliche Kräfte in der Welt aus: diejenige von Kannon - einer Gottheit mit weiblichen Attributen-, und diejenige der dämonischen Götter (kishin). ${ }^{26}$

Die dritte Charakteristik von Izumi Kyōkas Literatur ist seine extravagante und bildhafte, zuweilen geradezu, wie Izawa Jun festhält, "filmhafte" (eigateki) ${ }^{27}$ Sprache. ${ }^{28}$ Kyōka setzte diese in seiner Literatur gleichsam als lebendige, wirkende Kraft ein: “Die Kraft der Worte”, so soll Kyōka auf ein Papier, das kurz nach seinem Tod im Schubfach seines Schreibpultes gefunden wurde, vermerkt haben, "ist unermesslich. Verwende sie sorgfältig." 29 Wie Inouye herausstreicht, versuchte Kyoka durch seine figurative Sprache bewusst, das Gesichtsfeld des Erzählers zu begrenzen, da die Präsenz eines Allsehenden innerhalb des Textes den Zugang zu dem, was in Wirklichkeit gesehen wird, hindernd einschränke. Die Funktion der Sprache liegt somit nicht in der Beschreibung der äusseren Welt, sondern was zählt, ist das Wort als Bild und narratives Prinzip. ${ }^{30}$

Weitere Merkmale von Izumi Kyōkas Literatur sind, wie Kawakami Chiyoko herausstreicht, eine Obsession mit dem Heiligen, eine Romantisierung der Ausgestossenen, eine Faszination für das Groteske sowie eine

20 Inouye 1996: 34.

21 Siehe u.a. Cornyetz 1998, 1999; Poulton 1995a; Sasahara 1976; Tanaka 2006.

22 Poulton 1995a: 89.

23 Cornyetz 1998: 105.

24 Poulton 1995b: 420.

25 Poulton 1995b: 421.

26 Inouye 1996: 34.

27 Izawa 1949, zit. nach Miyoshi 1982: 13.

28 Hughes 2000: 60. Für Ausführungen zu Izumi Kyōkas Sprache siehe Inouye 1996.

29 Inouye 1996: 5.

30 Inouye 1996: 29. 
Liebhaberei für Volkserzählungen. ${ }^{31}$ Das "Dorf” - ein wiederkehrender Schauplatz in Kyōkas Literatur - bilde, so Kawakami, ein Teil der modernen Realität, dessen sich der Stadtmensch nicht bewusst sei. Kyōka eröffne dem urbanen Leser somit einen Blick auf eine Anderswelt, das unheimliche Andere inmitten der japanischen Moderne. ${ }^{32}$

Die hier übersetzte Kurzgeschichte Yōsoki 妖僧記 (Aufzeichnungen über einen unheimlichen Mönch) aus dem Jahr 1902 steht ganz im Zeichen der hier erläuterten Charakteristika von Kyōkas Literatur. Die Erzählung, zunächst in der Zeitung Kyūshu Nichinichi Shinbun erschienen, fand 1909 Aufnahme in den Sammelband Yanagibako 柳管 (Die Schachtel aus Weidenholz) und später in die Gesamtausgabe von Kyōkas Werken (Kyōka zenshū). Die Geschichte handelt von einem hässlichen Bettelmönch mit riesiger Nase, der in Kurokabe, einem abgelegenen Dorf in der Nähe von Kanazawa, Kyōkas Geburtsort, sein Unwesen treibt und um eine schöne Frau namens Otsū buhlt. Charakteristisch sind nicht nur die fantastischen und volkstümlichen Elemente, sondern auch hier spiegelt sich Kyōkas Sehnsucht nach seiner früh verstorbenen Mutter, verlagert auf die Figur der um den Tod ihrer Mutter trauernden Otsū. Yōsōki entlehnt zudem verschiedene Elemente aus früheren Erzählungen. So ist Kurokabe auch Schauplatz der gleichnamigen Erzählung Kurokabe 黒壁 (1894). Eine Figur mit riesiger Nase taucht zudem bereits in der Erzählung Seidan Jūnisō 政談十二社 (Politische Gespräche in Jūnisō, 1900-1901) auf. Die Idee eines Mönchs, der vor dem Grab der Mutter der Protagonistin im Schneidesitz sitzt (die Schlussszene von Yōsōki), ist ausserdem der Erzählung Nanamotozakura な〉もと桜 (sieben Kirschbäume, 1897) entlehnt. Die eigentliche Vorlage von Yōsōki bildet indes vermutlich das Manuskript Gama hōshi (Der Krötenmönch), verwahrt in der Keiō Gijuku Bibliothek. Jenes endet aber anders als die vorliegende Erzählung, nämlich damit, dass Otsū dem Mönch einen Spiegel, ein Andenken ihrer Mutter, vorhält, worauf der Mönch, erschrocken über sein eigenes Antlitz, flüchtet und fortan von seinen Avancen ablässt. ${ }^{33}$

Izumi Kyōkas bildhafte, gefühlsbetonte Sprache entfaltet sich auch in Yōsōki in vollendeter Form. So wird das Auftauchen des unheimlichen, grossnasigen Bettelmönchs in Nodayama mit dem Hervorwinden einer Made aus dem Morast verglichen, und das Bild, wie die Schönheit Otsū eines Abends nach einem Bad mit leicht über die Schultern geworfenem Gewand und lose gebundenem Gürtel auf der Veranda sitzt, wobei sich ihre "schneeweisse Brust gegen das Rot ihrer Hüfte” abhebt - Kyoka soll eine Fixierung auf die Farben rot und weiss gehabt

31 Kawakami 1999: 580.

32 Kawakami 1999: 581-582.

33 Muramatsu 1982: 75. 
haben ${ }^{34}$ - besticht durch eine einprägsame und wehmütige Ästhetik. Die Eigentümlichkeit von Kyōkas figurativer Sprache stellt den/die Übersetzer/in mancherorts allerdings vor Herausforderungen: So mussten einige extravagante poetische Bilder wie "von da an wurde die Nase sehr mächtig", "er entfloh hastig mit fliegender Nase" oder "sie weinte Tränen der Erinnerung" schweren Herzens einer in der deutschen Sprache etwas natürlicheren Formulierung weichen. Es wurde allerdings versucht, dem Original möglichst treu zu bleiben.

\section{2 Übersetzung}

\section{1}

Eine Meile vom Stadtrand von Kanazawa entfernt, im Distrikt Kaga, liegt die Ortschaft Kurokabe. ${ }^{35}$ Im ganzen Land erzählt man sich, dass dort Dämonen hausen. Vielleicht liegt dies an dem düsteren Wald hinter dem Berg Nodayama. Dort gibt es eine kleine eingefriedete Bergsiedlung, deren Zentrum ein Tempel bildet. In diesem leben Bergmönche, welche den dort aufgebahrten Marīci ${ }^{36}$ verehren. Die Siedlung besteht aus etwas mehr als dreissig Häusern mit vierzig bis fünfzig Einwohnern. Die Menschen, die sich dort versammeln, sind der irdischen Welt überdrüssig, haben sich von ihr abgewandt und verbringen nun gemächlich ihre müssigen Tage. So hört man zu jeder Tageszeit Gesang in harmonischer Abstimmung zum Klang von Flöten und Trommeln des Maibayashi-Tanzes, ${ }^{37}$ und auch mitten in der Nacht dringen unversehens die Klänge von Koto und Biwa leise in die schlafenden Ohren der Einwohner von Kanazawa.

Eines Jahres im Frühsommer begann in diesem Gebiet ein aussergewöhnlich widerwärtiger Bettelmönch herumzulungern. Es ist unbekannt, woher er kam. Ganz plötzlich tauchte er in Kurokabe auf, und fast gleich dem Anblick einer Made, die im Morast heranwächst, schien er sich aus der Natur herauszuwinden. Er war in jenem Jahr wohl um die 35 Jahre alt. Er hatte sich eine mausgraue, zerfetzte Priesterrobe umgebunden, die an ihm herunterhing und kaum zusammenhielt.

Sein Gesicht war furchtbar ausgemergelt, sein ganzer Körper schwarz und abgemagert, die Fingernägel lang, der Bart kurz, soweit also ein normaler

34 Inouye 1996: 7.

35 Ich bedanke mich bei Andrea Möser und Fynn Holm für ihren wertvollen Input bezüglich einiger Formulierungen in dieser Übersetzung.

36 Indischer Kriegsgott.

37 Musikstil mit Trommeln und Flöte; im Nō Theater oft von Tanz begleitet. 
Bettler. Sah man aber erst einmal seine Nase, so kam niemand umhin, über dieses sonderbare Spiel der Natur zutiefst erstaunt zu sein. Die Nase war gross und lang und in die Breite aufgeschwollen. Ihre Spitze war leicht krumm und glänzte rötlich. Da der Nasenrücken ausgeprägt war, erstreckte sich die Nase von Stirn bis zum Mundansatz und nahm so das ganze Gesicht ein. Die mageren Backen waren kaum vorhanden. Würde man mit der Handfläche die Nase des Bettelmönchs verdecken, verschwände sein gesamtes Gesicht. Von weitem sah er aus wie eine wandernde Nase mit einem Wanderstock.

Der Bettelmönch hielt diesen Stock stets fest in der Hand und liess ihn keinen Augenblick los. Der Stock war aus einem hakenförmigen, natürlich gewachsenen Stück Holz. Wenn er nichts zu tun hatte, ruhte er sich stehend aus, indem er seine Nase auf dessen gebogenen Teil abstützte und seine Hände auf Hüfthöhe hinter dem Rücken verschränkte. Kurz: so wie wenn wir uns auf einen Stuhl, setzen um uns auszuruhen, war für den Bettelmönch der Wanderstock der Stuhl für seine Nase.

Der Besitzer dieser aussergewöhnlichen Nase war zweifellos ein Bettler, doch heischte er niemals um Mitleid und hatte noch nie jemandem Geld oder Reis abverlangt. Wenn er Almosen bekam, nahm er diese mit grossmütiger Manier entgegen und spielte sich dabei auf wie ein Hohepriester, der eine Gabe in Empfang nimmt. Wenn eine Person sich über diesen Hochmut aufregte und beschloss, ihm weder Geld noch Essen zu geben, tat der Bettelmönch absichtlich so, als liesse ihn dies kalt, und er sah auch keineswegs hungrig aus.

In Kurokabe gab es nämlich so viele Kröten, dass sich seine Bewohner damit brüsten konnten, dass es im Sommer nicht eine einzige Mücke gab. Der Bettelmönch sammelte diese Kröten geschickt ein, zerriss sie in Stücke und verschlang sie, wobei er sich pro Abend mit einem guten Dutzend begnügte. Deshalb versteckte er sich tagsüber irgendwo, wurde von niemandem gesehen und wartete den Zeitpunkt ab, an dem die Kröten zur Abenddämmerung hervorkrochen. Dann erschien er wie aus heiterem Himmel, tauchte in der Düsterheit unter einem Vordach, bei einem Zaun oder einer Hecke auf, um dann wieder zu verschwinden, nachdem er seinen Hunger gestillt hatte.

\section{2}

Als vollkommenes Gegenbild zu diesem abscheulichen Krötenmönch lebte in Kurokabe eine Frau in blühendem Alter, schön wie eine Perle. Sie hiess Kiyokawa Otsū, war unverheiratet und hatte weder Eltern noch Geschwister. Im selben Haus wohnte ausser ihr nur noch eine Greisin, die ihr Dienstmädchen war. 
Otsū war die Tochter eines gewissen Herrn Kiyokawa, ein ehemaliger Lehensmann mit einem Lehen von $500 \mathrm{Koku}^{38}$ Reis. Bereits als Kind hatte sie ihren Vater verloren und im vorletzten Jahr ihre Mutter, so dass sie schliesslich ganz allein war. Es war ihr lästig, dass Bekannte sie dazu drängten, sich in eine Ehe zu begeben oder sich einen Bräutigam zu nehmen. Deshalb verliess sie am Ende des ersten Todestags ihrer Mutter ihr Haus in Kanazawa und zog im vergangenen Jahr hierher. Da sie ein grosses Vermögen besass und es ihr nicht an Kleidung und Nahrung mangelte, verbrachte sie ihre Tage in Luxus und Eleganz. Es fehlte ihr an nichts, jedoch wirkte ihr Gesicht stets wie ein von Wolken umhüllter Mond. Auch die Zeit vermochte nicht, die Tränen wegzuwischen, die ihr auch heute noch aus Trauer über den Tod ihrer Mutter über die Wangen flossen. Selbst Vergnügungen wie Lesen, Koto spielen oder die Schönheiten der Jahreszeiten vermochten ihren Trübsinn nicht zu lindern, sondern erweckten vielmehr schmerzliche Erinnerungen. Ihren einzigen Trost fand sie darin, deren Grabstätte in Nodayama zu pflegen und sich, ihre Mutter anrufend, weinend an ihr Grab zu klammern. Für Otsū war dieser Besuch des Grabes zum steten Tagewerk geworden.

Am 15. Juli, dem besonderen Tag des Seelenfestes, verliess Otsū in der abendlichen Kühle ihr Haus und begab sich alleine zum Grab der Mutter. In Nodayama gab es zwar viele Gräber, doch da sehr wenige Leute diese besuchten und es auch keine Mönche gab, welche diese pflegten, wucherte das Unkraut, die hölzernen Totentafeln waren umgekippt, die Grabhügel zerfallen und die Gräber unordentlich. Soweit das Auge reichte, herrschte furchtbare Trostlosigkeit.

Wie immer weinte Otsū dort von Erinnerungen überwältigt, brachte Blumen dar, entzündete Räucherstäbchen und umhegte die Mutter liebevoll, fast so als wäre diese anwesend, und sprach mit ihr mehr als zwei Stunden. Als Otsū nach Hause zurückkehrte, war der Weg bereits dunkel. Schnellen Schrittes eilte sie nach Kurokabe zurück. Mit einem Abstand von zwanzig Metern schlich ihr heimlich jener Bettelmönch hinterher. Er verfolgte Otsū nunmehr seit fast zehn Tagen. Wenn die Schönheit ausging, heftete er sich ständig wie ein Schatten an ihre Fersen. Sie wusste nichts davon, doch gab es Leute, die dies bemerkt hatten.

Auch an diesem Abend, nachdem er die schöne Frau bis zu ihrem Haus verfolgt hatte, stand der Bettelmönch neben den Wurzeln einer Zeder herum und ruhte sich aus, die Nase wie gewöhnlich auf seinen Stock gestützt. Da drang aus dem Innern der Hecke ein feiner Geruch hervor. Der Mönch blähte seine Nüstern, und als er heimlich hineinspähte, hatte die Schönheit vermutlich gerade gebadet, denn sie hatte ihr Gewand leicht über die Schultern geworfen, und da sie sich ungesehen wähnte, sass sie mit locker gebundenem Gürtel auf der Veranda. Ihre schneeweisse Brust hob sich gegen das Rot ihrer Hüfte ab. Der

38 Masseinheit für die Reisstipendien von Fürsten und Samurai. Ein Koku entspricht 180 Liter Reis. 
Krötenmönch betrachtete diese blendende Schönheit von allen Seiten, schüttelte seine Hände, stellte sich auf die Zehenspitzen und machte komische Gebärden gleich denen einer Marionette. Danach kehrte er unvermittelt auf dem Absatz um, machte überdies einen Umweg, ging in Richtung des geflochtenen Gartentors, lachte kurz auf und sprach, während er seine grosse Nase in den Garten hinein streckte: "Gib mir eine Schale warmen Reis."

Menschen, die den Bettelmönch noch nicht kannten, hätten, wären sie plötzlich auf diese Nase getroffen, zumindest laut aufgeschrien, aber die schöne Frau kannte ihn bereits. Da sie zudem gehört hatte, dass der Mönch ein verrückter Dummkopf ohne gesunden Menschenverstand sei, verzog Otsū keine Miene und fuhr damit fort, ihr beim Baden zerzaustes Haar vor einem Spiegel glattzukämmen.

Der Krötenmönch neigte seine Nase zweifelnd und betrachtete Otsūs Tätigkeiten von allen Seiten. Er fragte mit komischer Stimme, auf den Spiegel in der Hand der Schönheit zeigend: "Was ist das?” Otsū schaute flüchtig zurück und antwortete, sich über seine komische Frage wundernd, nur: "Ein Spiegel.” Der Dummkopf fragte abermals: "Wozu dient es?" Darauf antwortete sie: "Es ist ein Gegenstand, um sein Abbild zu sehen. Wollt Ihr, ehrenwerter Mönch, ebenfalls Eure Nase abgebildet sehen?" und streckte ihm den Spiegel entgegen. Der Krötenmönch sprang zurück, und sichtlich verängstigt entfloh er hastig, mit auf und ab tanzender Nase.

Als diese Geschichte herumerzählt wurde, erkannten die Bewohner von Kurokabe den Charakter des Krötenmönchs. Zudem bemerkten sie, dass der Bettelmönch absurde Gefühle gegenüber Otsū hegte und waren sich mehr und mehr im Klaren darüber, dass er ein Dummkopf war.

Der Bettelmönch hingegen murmelte, während er davonlief, nachdem ihm der Spiegel gezeigt worden war, im Stillen die folgenden Worte: "Ich schwöre mir, bis die Welt endet, werde ich nie mehr in einen Spiegel blicken, jawohl, auf keinen Fall werde ich je wieder in einen Spiegel blicken. Nein, nicht nur das, ich muss selbst den Namen des Dings, das Spiegel genannt wird und das mir nun wieder in Erinnerung gerufen wurde, möglichst vergessen.”

\section{3}

Die Personen, die bemerkten, dass der Krötenmönch ein Interesse an Otsū hatte, erzählten dies Otsūs alter Dienerin, spotteten und warnten. Auch die Dorfbewohner, die sich jeden Abend auf einer Bank draussen versammelten, um sich abzukühlen, lästerten laut über den Krötenmönch. Sie vereinbarten, dass demjenigen, der die Wohnstätte des Bettelmönchs und seine Herkunft herausfinden würde, als Wettbetrag ein Yen ausgehändigt würde. 
Eines Abends besuchte Otsū wie üblich das Grab in Nodayama. Als sie zurückkehrte, war die Sonne bereits untergegangen. Nachdem sie das Licht angezündet hatte, öffnete sie das Fenster, setzte sich mit dem Rücken zum Lotusteich vor dem Haus an ihren Schreibtisch und schrieb, während sie sich abkühlte, zur Totenandacht der verstorbenen Mutter das Lotos-Sutra ab. Daneben führte die alte Frau eifrig den Nähfaden. In diesem Augenblick kam der Krötenmönch plötzlich aus dem Nichts herbei, und kaum erblickte er Otsūs Gestalt, verengte er die Augen, leckte sich die Lippen und war wie verzaubert. Wahrscheinlich dachte der Bettelmönch, dass die Schönheit duftete, denn er blähte seine Nase, blickte durch das Gitterfenster, steckte die Nase rücksichtslos herein und versuchte, Otsūs Wange zu streifen. Erschrocken über den unerwarteten Gast wich Otsū mit einem Aufschrei zurück, und da die Situation so komisch war, prustete die alte Frau gleichzeitig los, ohne Zeit für Tadel zu finden.

Der Krötenmönch schien wohl fälschlicherweise $\mathrm{zu}$ denken, dass er ihr Wohlwollen erhalten habe, denn er strahlte vor Freude über das ganze Gesicht, lachte nickend und plapperte ohne Unterlass. Doch Otsū, die ihn schon lange hasste, blieb kühl und schenkte ihm keinerlei Beachtung. Natürlich ignorierte ihn auch die alte Frau, doch die Nase quatschte weiterhin unverschämt unnützes Zeug. Da sie seine Belästigungen nicht mehr ertragen konnte, befahl sie ihm mit der Hand: “Geh weg!” Doch da die Nase sich einfach nicht zurückzog, verlor die alte Frau die Geduld und stiess verärgert die Nadelspitze in ihrer Hand in seine Nasenspitze.

Da nun dem erbärmlichen Bettelmönch solcherart ein schmerzhafter Hieb versetzt worden war, rief er: "Autsch!”, bog seinen Körper zurück und lief, während er sich Speichel auf seine Nase strich und diese rieb, hastig davon. Doch nach etwa hundert Metern hielt er plötzlich inne, blickte zurück und durchbohrte mit seinen Blicken das Lampenlicht auf der gegenüberliegenden Seite des Lotusteichs. Sein Blick war unheimlich und voller Hass. In diesem Moment erhob der Bettelmönch seinen Stock und rief entschlossen: "Wenn man bei der Wahl der Mittel nicht wählerisch wäre, welchen Wunsch könnte man dann nicht erfüllen?” Darauf trommelte er mit dem Stock fest auf die Erde, zog den Kopf ein, stützte sich auf seinen Stock und ging langsamen Schrittes von dannen. Eine alte Frau schlich ihm heimlich nach. Es war das alte Dienstmädchen jener Otsū, die nach dem noch niemandem bekannten Wohnort des Bettelmönchs suchte, um den Einsatz zu erhalten, der bei der Wette auf der kühlen Abendbank geschlossen worden war. Sie hatte nicht auf Otsūs Einwände gehört und war ihm gefolgt. Mit angehaltenem Atem schlich sie ihm nach. Der Dummkopf schien sie aber nicht wahrzunehmen, stützte sich auf seinen Stock und lief, seine Beine nachziehend, dahin. 
Er entfernte sich vom Dorf. Als er etwa zweihundert Meter in nördliche Richtung vorangeschritten war, öffneten sich die Berge zu einem Tal hin. Dort ragte eine Felswand empor, deren steiles Gefälle wie eine einzige Mauer aussah. Im Tal zwischen den Bergen warfen dicht aneinandergereihte Kiefern und Zedern lange Schatten. Da die Gräser und Bäume eine lackschwarze Farbe hatten, wurde das Tal Kurokabe, die schwarze Mauer, genannt. In einer Höhle in der Mitte dieses Tals wurde besagter Marīcī verehrt.

Die weit in die Tiefe abfallende Bergschlucht war selbst am hellichten Tag eine dunkle, andere Welt. Bei Tag und bei Nacht war sie dunstverhangen, so als würde es unter den Füssen nieseln. Ein Gebirgsbach flüsterte Teufelsworte und eine dämonisch wimmernde Atmosphäre überfiel die Menschen. Es war von beispielsloser Schrecklichkeit.

Die alte Frau, die nie gedacht hätte, dass er dort wohnen würde, überkam eine unerträgliche Angst. Sie fühlte, als würden böse Geister eine Reihe bilden und ihr den Weg versperren. Trotz ihrer Gier konnte sie keinen Schritt weitergehen und blieb verängstigt wie versteinert stehen. In diesem Moment wandten sich zwei Lichtpunkte, die wie Glühwürmchen aussahen, plötzlich nach ihr um und donnerten: "Wer bist du?" Unter dem Stock, den der Krötenmönch hochhielt, duckte sich die alte Frau erschrocken.

Der Krötenmönch blickte von oben auf sie herab und sagte höhnisch lachend: "Hahaha, ja, tatsächlich, du bist es, der unverschämte Mensch, der mich im Gesicht verletzt hat. Weisst du nicht, dass es so etwas wie Rache gibt?” Als sie dies hörte, wurde der alten Frau bis ins Innerste ihres Herzens kalt, als wäre ihr ganzer Körper zu einem Eiszapfen geworden. Sie sprach mit elender Stimme: "Bitte, lasst mir wenigstens mein Leben!” und zitterte dabei heftig.

\section{4}

Derweil hatte der Krötenmönch die alte Frau zu Tode erschreckt. Er sprach grob zu ihr: "Alte, lass mich durch Vermittlung mit deiner Herrin meine Rachsucht besänftigen. Wenn Du mir nicht ein lebendiges Opfer darbringst, dann wird nicht nur Otsū, sondern auch Dir etwas Schlimmes widerfahren. Du musst auf jeden Fall zwischen uns vermitteln. Bis dahin werde ich dein Leben verschonen, Du seniles, dem Tod nun glücklich entkommenes altes Weib!” Sie fühlte, als würde ein heftiger Wind wehen und die Erde aufwirbeln. Als sie eingeschüchtert den Kopf hob, war der Krötenmönch bereits hinabgestiegen und im Dunst verschwunden, so als hätte er seinen Körper auf den Grund des Tales fallen lassen.

Als sie leichenblass und heilfroh, mit dem Leben davongekommen $\mathrm{zu}$ sein, davonfloh und heimkehrte, wurde sie, sobald sie erblickt wurde, sofort von zwei bis 
drei Männern, die immer noch auf der nun kühl gewordenen Bank sassen, von rechts und links bestürmt: "Du hast wohl Nachforschungen betrieben? Wo wohnt der Krötenmönch denn nun?” Da hob sie ihre Faust vor der Nase und antwortete mit zittriger Stimme: "Es war furchtbar, aber wenigstens habe ich überlebt.” Daraufhin berichtete sie ihre Erlebnisse und schloss mit den Worten, der Krötenmönch sei, so könne man sagen, wohl eher dämonisch als ein Idiot oder ein Wahnsinniger. Und wenn nicht, dann müsse er doch zumindest ein Hexenmeister sein.

Von da an wurde der Krötenmönch mit der grossen Nase sehr mächtig, erschreckte die Leute insgeheim und herrschte über ganz Kurokabe. Dies, weil Jung und Alt den dämonischen Mönch gewöhnlich ängstlich mieden und versuchten, sich von ihm fernzuhalten. Wer wünschte sich nicht, ein unheimlicher Stern kehre in den Himmel zurück und verschwände aus der Sicht?

Am meisten wünschten sich dies die Kröten und Kiyokawa Otsū. Denn die schöne Frau hatte zufällig bei den Gesprächen auf der kühlen Abendbank mitgehört wie jemand sagte, der vielgehasste und unerträgliche Bettelmönch sei aufgrund der Kröten und Otsū in Kurokabe aufgetaucht. Jedes Mal, wenn sie daran dachte, betete sie aus lauter Furcht vor dessen fürchterlichen Anhänglichkeit ihre verstorbene Mutter an und flehte, diese möge den sich ständig an ihre Fersen heftenden Teufel vertreiben. Otsū glaubte in ihrem Herzen, dass ihre verstorbene Mutter, so als wäre sie immer noch leibhaftig da, wie in ihrer Kindheit ihren Wunsch bestimmt erhören würde, wenn sie nur darum bäte.

Doch was konnte man nun noch dagegen ausrichten? Schliesslich wurde Otsū aus dem Mund der alten Frau verkündet, es sei unvermeidlich, dass sie das Opfer des Bettelmönchs werde. Die alte Frau, die dem Kurokabe einen Besuch abstattenden Krötenmönch den grössten Glauben schenkte und an seiner Zauberkraft keinesfalls zweifelte, hatte zwar tagtäglich gezögert, Otsū als Vermittlerin auszurichten, was er ihr vor Kurzem gedroht hatte, nämlich, dass es ihr nicht zum Guten gereichen würde, wenn sie ihm die schöne Frau nicht als Tribut zahlte. Doch da sie von dem Bettelmönch stark dazu gedrängt wurde, war es schliesslich unvermeidlich geworden, dies Otsū auszurichten.

Da Otsū dies jedoch schon ahnte, war sie nicht so überrascht wie die alte Frau gedacht hatte. Die Schönheit belehrte die alte Frau kühl: "Überlege Dir zuerst, wie meine Mutter urteilen würde, wenn sie noch auf dieser Welt wäre. Sie würde bestimmt nicht sagen, ich solle die Frau dieses Bettlers werden.” Auf diese Worte wusste die alte Frau keine Antwort, doch da sie furchtbar abergläubisch war, nahm sie die Drohung des Bettelmönchs ernst und dachte, es handle sich um eine Angelegenheit, bei der es um Leben und Tod ginge. Sie sagte eifrig: "Da dieser Kerl ein Hexenmeister mit grossen übernatürlichen Kräften ist, ist es schwer zu ermessen, was er im Schilde führt. Dennoch sage ich nicht, dass Ihr der Nase Folge leisten solltet. Habt Ihr denn keine gute Idee?” Daraufhin antwortete jene 
kühl: "Nein, ich habe keine Idee. Doch was auch immer geschieht, ich werde keinesfalls etwas tun, das dem Willen meiner Mutter widerspricht."

Auf diese ausdrückliche Verweigerung wusste die alte Frau nicht zu reagieren und geriet in Bedrängnis. Sie schlug sich aus unerfindlichen Gründen auf ihr Knie und sagte: "Es gibt auf dieser Welt niemanden, der durch und durch unnachgiebig und furchtbar ist. Um der schwierigen Aufgabe der Nase zu entkommen, müssen wir von unserer Seite ebenfalls eine ausgesprochen schwierige Aufgabe vorbringen, und wir tun am besten daran, mit ihm zu vereinbaren, dass Ihr seinem Wunsch Folge leistet, wenn er etwas Bestimmtes erfüllt. Es gibt eine alte Erzählung von einer Schlange, die sich in die Frau eines Kriegers verliebt und ihr hartnäckig nachstellt. Ihr Mann ist eine mit Weisheit ausgestattete Person. Er verspricht der Schlange trügerisch: 'Wenn du drei grosse Adler fängst und mir diese übergibst, dann gebe ich dir meine Frau.' Die Schlange stimmt zu, kämpft mit den Adlern und wird dabei vernichtet. Doch wenn wir nun unüberlegt den Kopf eines Adlers fordern, dann ist nicht auszuschliessen, dass sich dieser furchtbare Hexenmeister wappnet. Deshalb habe ich mir folgendes überlegt: Ich teile dem Bettelmönch mit, dass Ihr ihm Folgendes ausrichtet: 'Ich bin mit Eurem Vorschlag einverstanden, aber ich brauche die Zustimmung meiner Eltern. Falls meine Mutter einwilligt, dann werde ich jederzeit Eure Frau werden. Geht nun und fragt zunächst meine Mutter.' Da Eure Mutter natürlich bereits nicht mehr auf dieser Welt ist, ist dies ein unmögliches Versprechen. Und da ein unmögliches Versprechen nicht eingehalten werden kann, wird bestimmt selbst diese Nase schwach und schliesslich aufgeben. Macht Euch also keine Sorgen.” Da die Worte der ihr solcherart zuredenden alten Frau vernünftig klangen, stimmte Otsū gedankenlos zu.

Nachdem die alte Frau dies dem Krötenmönch nun so mitgeteilt hatte, verschwand die Nase aus Kurokabe. Da rühmte sich die Alte vor Otsū und den Dorfbewohnern auf der kühlen Bank, dass alles wie geplant gelaufen war.

Jedoch, als Otsū an diesem Abend wiederum zum Grab auf dem Friedhof von Nodayama pilgerte, sah sie den verschwunden geglaubten Krötenmönch im Lotussitz vor der Grabstätte der Mutter sitzen und wurde leichenblass.

\section{Bibliographie}

Cornyetz, Nina (1998): “Izumi Kyōka’s Jouissance: Dangerous Women and Deadly Words”. In: Proceedings of the Midwest Assocation for Japanese Literary Studies (PMAJLS) 4

(The New Historicism and Japanese Literary Studies), 104-119.

Cornyetz, Nina (1999): Dangerous Women, Deadly Words. Phallic Fantasy and Modernity in

Three Japanese Writers. Stanford: Stanford University Press.

Hughes, Henry J. (2000): “Familiarity of the Strange: Japan's Gothic Tradition”. Criticism 42.1: 59-89. 
Inouye, Charles Shirō (1996): “Izumi Kyōka and Language”. Harvard Journal of Asiatic Studies 56.1: 5-34.

Izawa, Jun 井沢淳 (1949): “Uta andon’ no okeru eigateki hyōgen”『歌行灯』における映画的表現. Kokubungaku kaishaku to kanshō 国文学解釈と鑑賞 14.5: 80-82.

Izumi, Kyōka 泉鏡花 (1942 [1902]): “Yōsōki” 妖僧記. In: Kyōka zenshū 鏡花全集, Bd. 7. Tōkyō: Iwanami Shoten, 171-182.

Izumi, Kyoka 泉鏡花 (1942 [1909]): “Bungei wa kanjō no sanbutsu nari” 文芸は感情の産物なり. In: Kyōka zenshū 鏡花全集, Bd. 28. Tōkyō: Iwanami Shoten, 723-727.

Kawakami, Chiyoko (1999): “The Metropolitan Uncanny in the Works of Izumi Kyōka: A CounterDiscourse on Japan's Modernization”. Harvard Journal of Asiatic Studies 59.2: 559-583.

Keene, Donald (1984): Dawn to the West. Japanese Literature of the Modern Era, Bd. 1. New York: Holt, Rinehart and Winston.

Mishima, Yukio 三島由紀夫 (1975): “Bunshō tokuhon” 文章読本. In: Mishima Yukio zenshū 三島由紀夫全集, Bd. 28. Tōkyō: Shinchōsha, 414-564.

Mishima, Yukio 三島由紀夫 (1976): “Kaisetsu, Nihon no bungaku 4: Ozaki Kōyō, Izumi Kyōka” 解説日本の文学4: 尾崎紅葉, 泉鏡花. In: Mishima Yukio zenshū 三島由紀夫全集, Bd. 33. Tōkyō: Shinchōsha, 553-555.

Miyoshi, Yukio 三好行雄 (1980): “Izumi Kyōka ni okeru ‘kyokō’ no imi” 泉鏡花における「虚構」 の意味. In: Nihon bungaku kenkyū shiryō sōsho kankōkai 日本文学研究資料叢書 (Hg): Izumi Kyōka 泉鏡花. Tōkyō: Yūseidō, 8-19.

Muramatsu, Sadakata 村松定孝 (1982): Izumi Kyōka jiten 泉鏡花事典. Tōkyō: Yūseidō.

Poulton, Mark Cody (1995a): "Metamorphosis: Fantasy and Animism in Izumi Kyōka”. Japan Review 6: 71-92.

Poulton, Mark Cody (1995b): “Onna Keizu/Oni Keizu: Izumi Kyka’s Iconology of Women”. In: The Force of Vision. Edited by Earl Miner and Haga Toru (The Organization for the Publication of the ICLA '91 Tokyo Proceedings; 6), 420-429.

Sasahara, Nobuo 笠原伸夫 (1976): Izumi Kyōka: Bi to erosu no kōzō 泉鏡花: 美とエロスの構造. Tōkyō: Shibundō.

Sasahara, Nobuo 笠原伸夫 (1984): “Izumi Kyōka” 泉鏡花. In: Nihon daihyakka zensho (Nipponica）日本大百科全書（ニッポニカ）. Tōkyō: Shōgakukan. https://japanknowledge.com/ lib/display/?lid=1001000021084 (letzter Aufruf 1.2.2019).

Tanaka, Takako 田中貴子 (2006): Kyōka to kaiyi 鏡花と怪異. Tōkyō: Heibonsha.

Tanizaki, Jun’ichirō 谷崎潤一郎 (1968): “Junsui ni ‘Nihonteki’ na ‘Kyōka sekai”” 純粋に「日本的」 な「鏡花世界」. In: Tanizaki Jun’ichirō zenshū 谷崎潤一郎全集, Bd. 22. Tōkyō: Chūō Kōronsha, 336-338.

Waki, Akiko 脇 明子 (1974): Gensō no ronri: Izumi Kyoka no sekai 幻想の論理: 泉鏡花の世界. Tōkyō: Kōdansha. (Kōdansha Gendai Shinsho).

Waki, Akiko 脇明子 (1980): “Yume gatari: Izumi Kyōka ni okeru sōzōryoku no muka” 夢がたり: 泉鏡花における想像力の夢化. In: Nihon bungaku kenkyū shiryō sōsho kankōkai 日本文学 研究資料叢書 (Hg): Izumi Kyōka 泉鏡花. Tōkyō: Yūseidō, 8-19. 